RELATO DE CASO

\title{
SÍNDROME DE TURNER, TIREOIDITE DE HASHIMOTO E DOENÇA DE CROHN EM IRMÃS: RELATO DE CASO
}
TURNER SYNDROME, HASHIMOTO'S THYROIDITIS AND CROHN'S DISEASE IN SISTERS: CASE REPORT

\author{
Carlos Alberto Rodrigues Junior ${ }^{1}$, Lara Luiz da Silveira Duarte ${ }^{1}$, Marcelo \\ Henrique Menezes ${ }^{1}$, Rodrigo Cesar Menezes ${ }^{2}$, Ana Paula de Santana ${ }^{3}$, \\ Rodolfo Rezende das Neves ${ }^{4}$, Fellipe Camargo Ferreira Dias ${ }^{5}$, Cerize \\ Rodrigues Lima Cardoso ${ }^{6}$, Dark Luzia dos Santos Neto ${ }^{6}$, Greice de Cassia \\ Souza Oliveira ${ }^{7}$, Patrícia Bastos Amorim ${ }^{8}$.
}

\section{@ ACESSO LIVRE}

Citação: Rodrigues Junior CA, Duarte LLS, Menezes MH, Menezes RC, Santana AP, Neves RR, Dias FCF, Cardoso CRL, Santos Neto DL, Oliveira GCS, Amorim PB (2017) Síndrome de Turner, Tireoidite de Hashimoto e Doença de Crohn em Irmãs: Relato de Caso. Revista de Patologia do Tocantins, 4(4): 29-33.

Instituição: ${ }^{1}$ Graduando em Medicina, Universidade Federal do Tocantins, Tocantins, Brasil. ${ }^{2}$ Graduando em Medicina, Universidade de Rio Verde, Goiás, Brasil. ${ }^{3}$ Graduando em Medicina, Centro Universitário UnirG, Tocantins, Brasil ${ }^{4}$ Médico, Universidade Federal do Tocantins, Tocantins, Brasil. ${ }^{5}$ Médico, Pósgraduando em Educação em Ciências e Saúde, Universidade Federal do Tocantins, Tocantins, Brasil. ${ }^{6}$ Pós-graduanda em Ciências da Saúde, Universidade Federal do Tocantins, Tocantins, Brasil. ${ }^{7}$ Gastropediatra, Hospital Infantil Público de Palmas, Tocantins, Brasil. ${ }^{8}$ Endocrinopediatra, Docente, Universidade Federal do Tocantins, Tocantins, Brasil.

Autor correspondente: Carlos Alberto Rodrigues Junior; carlos.rouft@gmail.com

Editor: Guedes V. R. Medicina, Universidade Federal do Tocantins, Brasil.

Publicado: 01 de dezembro de 2017.

Direitos Autorais: (C) 2017 Rodrigues Junior et al. Este é um artigo de acesso aberto que permite o uso, a distribuição e a reprodução sem restrições em qualquer meio, desde que o autor original e a fonte sejam creditados.

Conflito de interesses: os autores declararam que não existem conflitos de interesses.

\section{RESUMO}

A Síndrome de Turner é caracterizada citogeneticamente pela presença de um cromossomo $\mathrm{X}$ e perda total ou parcial do segundo cromossomo sexual, ocorrendo em aproximadamente 1:2.130 nascidos vivos do sexo feminino. A Tireoidite de Hashimoto é um distúrbio autoimune decorrente de resposta imune anormal à glândula tireoide, tanto do ponto de vista humoral como celular. A doença de Crohn é uma doença crônica recidivante que afeta todas as partes do tubo digestivo, sendo multifatorial, em que fatores de ordem genética, imunológica e ambientais têm uma relevância preponderante no início e na perpetuação da lesão tecidual imunomediada. $O$ objetivo deste trabalho foi relatar o caso de três pacientes de uma mesma família. Caso 1, primeira gemelar (dizigótica), 8 anos de idade, sexo feminino, com diagnóstico de Tireoidite de Hashimoto. Caso 2 (segunda gemelar), sexo feminino, com o diagnóstico de Síndrome de Turner. Caso 3 (primogênita), sexo feminino, com diagnóstico de Doença de Crohn. Duas filhas de um casal hígido manifestaram doenças autoimunes, as quais têm incidência aumentada na síndrome de Turner. Doenças endócrinas autoimunes possuem mecanismos complexos com a participação de vários fatores, como a susceptibilidade genética, eventos ambientais e resposta autoimune, porém, neste caso, não se encontrou história familiar positiva além da geração estudada. Outros estudos familiares, como este que se propõe, com múltiplos membros acometidos, poderão identificar associações cada vez mais consistentes entre essas doenças, além daquelas ainda não suspeitadas.

Palavras-chave: Síndrome de Turner, Tireoidite de Hashimoto, Doença de Crohn, Hereditariedade.

\begin{abstract}
Turner syndrome is characterized cytogenetically by the presence of an X chromosome and total or partial loss of the second sex chromosome, occurring in approximately 1:2,130 live female births. Hashimoto's Thyroiditis is an autoimmune disorder resulting from an abnormal immune response to the thyroid gland, both from a humoral and cellular point of view. Crohn's disease is a chronic recurrent disease that affects all parts of the digestive tract, being multifactorial, in which genetic, immunological and environmental factors have a preponderant relevance at the beginning and the perpetuation of immune-mediated tissue injury. The objective of this study was to report the case of three patients from the same family. Case 1, first twin (dizygotic), 8 years old, female, with diagnosis of Hashimoto's Thyroiditis. Case 2 (second twin), female, with the diagnosis of Turner Syndrome. Case 3 (first-born), female, with diagnosis of Crohn's Disease. Two daughters of a healthy couple have manifested autoimmune diseases, which have an increased incidence in Turner syndrome. Autoimmune endocrine diseases have complex mechanisms with the participation of several factors, such as genetic susceptibility, environmental events and autoimmune response, but in this case, no positive family history was found beyond the generation studied. Other family studies, such as this one proposed, with multiple affected members, will be able to identify increasingly consistent associations between these diseases, in addition to those not yet suspected.

Keywords: Turner syndrome, Hashimoto's thyroiditis, Crohn's disease, Heredity.
\end{abstract}


INTRODUÇÃO

A Síndrome de Turner (ST) é caracterizada citogeneticamente pela presença de um cromossomo $X$ e perda total ou parcial do segundo cromossomo sexual. É geralmente um evento esporádico dentro de uma família e seu risco de recorrência na irmandade é muito baixo. Em cerca de $80 \%$ dos casos, as pacientes com ST herdaram seu cromossomo X normal da mãe, ou seja, houve falha na meiose paterna ou o cromossomo sexual paterno era estruturalmente anômalo ${ }^{1}$, ocorrendo em aproximadamente 1:2.130 nascidos vivos do sexo feminino ${ }^{2}$.

O fenótipo da ST é altamente variável. São sinais clínicos relevantes a baixa estatura e a disgenesia gonadal, além de diversos dismorfismos, malformações, patologias adquiridas, metabólicas, ósseas e autoimunes, tais como: tiroidite, doença celíaca, doença inflamatória intestinal, diabetes tipo 1, doença de Graves, colite ulcerativa, doença de Crohn, artrite reumatóide juvenil, psoríase, alopecia e vitiligo, entre as quais destaca-se a doença tireoidea autoimune, que parece ter um amplo espectro, de hipertireoidismo a hipotireoidismo ${ }^{3,4}$. Os ovários de pacientes com ST podem degenerar durante a vida fetal, na infância ou no início da idade adulta, apresentando-se com infertilidade, amenorreia primária e menarca espontânea em diferentes proporções ${ }^{5}$. Embora a infertilidade seja um importante desafio que afeta mulheres com ST, o desenvolvimento sexual também é uma questão que deve ser avaliada, principalmente durante a adolescência ${ }^{6}$.

A Tireoidite de Hashimoto (TH) é um distúrbio autoimune decorrente de resposta imune anormal à glândula tireoide, tanto do ponto de vista humoral como celular. Assim como na doença de Graves (DG), a demonstração de que parentes de casos índices têm maior probabilidade para desenvolverem a doença indica a existência de predisposição genética $^{7}$. Segundo Medeiros et al. (2007), a causa das alterações variáveis, transitórias e recorrentes nas concentrações de TSH e hormônios tireóideos observadas na ST, e muitas vezes iniciadas precocemente na vida dessas pacientes, pode ser um processo autoimune crônico e subclínico da tireoide, sendo a Ultrassonografia (USG) o método mais sensível para sua detecção, principalmente na faixa etária pediátrica. A prevalência de tiroidite de Hashimoto em pacientes com ST varia de $13,3 \%$ a $55 \%$, de acordo com os critérios utilizados para o diagnóstico, a faixa etária coberta, e a técnica utilizada para medir anticorpos ${ }^{8}$.

A maioria das doenças endócrinas autoimunes apresentam marcadores HLA (Human Leukocyte Antigen) de susceptibilidade semelhantes nas populações caucasianas, usualmente incluindo os grupos de alelos HLA-DRB $1 * 03 / * 04 \mathrm{e}$ DQB $1 * 02 / 0302$. Embora os antígenos e alelos de histocompatibilidade estejam envolvidos na patogenia dessas endocrinopatias, outros fatores genéticos, alguns já identificados, também estão envolvidos ${ }^{7}$. Muitos estudos acerca das associações entre os alelos HLA e as endocrinopatias autoimunes se concentram no diabetes mellitus tipo 1 (DM1) e doença de Graves (DG), mas outras endocrinopatias também têm sido estudadas, incluindo a $\mathrm{TH}$, a doença de Addison, a síndrome poli glandular autoimune e a insuficiência ovariana prematura ${ }^{7}$.
Já as doenças inflamatórias intestinais (DII), classicamente conhecidas como retocolite ulcerativa inespecífica (RCUI) e doença de Crohn (DC) são processos inflamatórios crônicos que cursam de maneira imprevisível com períodos de atividade e remissão variáveis; apesar de sintomatologia clínica semelhantes, são consideradas doenças distintas ${ }^{9}$. A doença inflamatória intestinal (DII) é uma doença crônica recidivante que afeta todas as partes do tubo digestivo, é multifatorial, em que fatores de ordem genética, imunológica e ambientais têm uma relevância preponderante no início e na perpetuação da lesão tecidual imunomediada. Embora ainda hoje não se conheça com clareza a sequência de eventos que desencadeiam a DII, a célula epitelial, as bactérias e a resposta imunológica inata parecem estar intrincadamente envolvidas ${ }^{10}$.

A DC é um tipo de DII, transmural, de natureza sistêmica e etiologia desconhecida, que pode afetar qualquer parte do tubo digestivo, estendendo-se da boca ao ânus ${ }^{11,12}$. Sua incidência é de cerca de dois casos/100.000 habitantes e a prevalência é de 50 casos/100.000 com variação geográfica e étnica, mais comumente encontrada em populações caucasianas. Acomete mais mulheres do que homens, apresentando um pico aos 20 anos e outro entre os $50-70$ $\operatorname{anos}^{13}$.

\section{APRESENTAÇÃO DOS CASOS}

Caso 1, primeira gemelar (dizigótica), 8 anos de idade, sexo feminino, mãe com queixas de emagrecimento sem anorexia, taquicardia, irritabilidade, agitação e diminuição do rendimento escolar. Antecedentes pessoais: nasceu de parto cesáreo, 35 semanas, peso de nascimento (PN): $2750 \mathrm{~g}$, estatura (E): $49 \mathrm{~cm}$. Ao exame físico, apresentou desenvolvimento neuropsicomotor (DNPM) adequado. Exames complementares: USG: tireoide aumentada sem nódulos ou cistos, inferindo doença difusa; hormônio tireoestimulante $(\mathrm{TSH})<0,01 \mu \mathrm{UI} / \mathrm{mL}$; tiroxina (T4) livre: 0,99 $\mu \mathrm{g} / \mathrm{dL}$; triiodotironina (T3) total: $123 \mu \mathrm{g} / \mathrm{dL}$; anticorpo antitireoperoxidase (anti-TPO) positivo; anticorpo antitireoglobulina (anti-TG) negativo; indicando o diagnóstico de Tireoidite de Hashimoto.

Caso 2 (segunda gemelar), sexo feminino, foi referenciada ao endócrino pediatria por baixa estatura aos 2 anos e 10 meses. Ao exame físico, apresentou DNPM adequado, estágio puberal: M1P1; tireoide sem alterações. Antecedentes Pessoais: nasceu de parto cesáreo, com 35 semanas; PN: $2270 \mathrm{~g}$; E: $44 \mathrm{~cm}$. Exames complementares: Idade óssea de 3 anos; TSH: 1,4 $\mu \mathrm{Ul} / \mathrm{mL}$; T4 livre: 1,0 ng/dL; ecocardiograma e USG de rins e vias urinárias sem alteração.

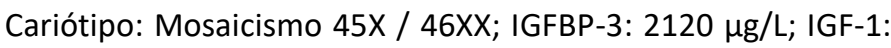
$52,9 \mu \mathrm{g} / \mathrm{L}$; indicando o diagnóstico de Síndrome de Turner.

Caso 3 (primogênita), sexo feminino, foi referenciada ao gastropediatra aos 11 anos de idade com queixa de diarreia intermitente há cerca de 1 ano e 6 meses. Mãe referiu diarreia com duração de até uma semana sem sangue ou muco. Ao exame físico, apresentou DNPM adequado. Antecedentes Pessoais: nasceu de parto cesáreo, a termo, sem intercorrências; PN: $3750 \mathrm{~g}$; E: $49 \mathrm{~cm}$; apresenta relato de constipação intestinal aos 12 meses de vida; internação aos 4 anos por pneumonia; aleitamento materno até 1 a $11 \mathrm{~m}$, 
sendo exclusivo até os 6 meses de vida; ingestão alimentar adequada, com frutas e verduras. Exames complementares: velocidade de hemossedimentação (VHS): $32 \mathrm{~mm} / \mathrm{h}$; IgA: 200 $\mathrm{U} / \mathrm{mL}$; Albumina sérica: $3,74 \mathrm{~g} / \mathrm{dL}$; pANCA positivo (1:40); cANCA negativo; ASCA IgG: 9,59 U/mL; ASCA IgA: 16,64 U/mL; pesquisa de sangue oculto nas fezes (PSOF) positivo; fator antinuclear (FAN) positivo (1/320); Colonoscopia: cólons e íleo terminal com presença de úlceras recobertas por fibrina, mucosa friável, intercaladas com áreas normais; Biópsia: íleo e cólon transverso com atividade inflamatória moderada e reto com atividade inflamatória leve; História Familiar: Pais hígidos. Estabelecido o diagnóstico de Doença de Crohn.

\section{DISCUSSÃO}

\section{Síndrome de Turner}

A ST também está associada a algumas anomalias médicas, como anormalidades cardiovasculares e renais, distúrbios da tireoide, perda de audição, hipertensão arterial, osteoporose e infertilidade ${ }^{14}$. Além disso, sabe-se que a autoimunidade está aumentada nesta síndrome ${ }^{15,16}$.

Vários genes localizados no cromossomo $X$ demonstraram ter possíveis funções reguladoras imunes. De nota, FOXP3 (Xp11.23, http://omim.org/entry/300292) desempenha um papel crucial para a função de $\mathrm{T}$ cells regulatórios naturais; as deleções neste gene causam síndrome de X-linked (IPEX) caracterizada por desregulação imune, poliendocrinopatia e enteropatia ${ }^{17}$.

Um estudo populacional dinamarquês com doentes com ST demonstrou que o risco de mortalidade por doenças endócrinas e metabólicas se encontra aumentado à custa da elevada frequência de diabetes; verificou ainda que a diabetes contribuiu para a mortalidade, mesmo quando não constituiu a verdadeira causa de morte ${ }^{18}$.

Portanto, estes estudos constituíram um passo significativo na compreensão da base genética destas alterações metabólicas, aparentemente mais prevalentes nas doentes com isocromossoma Xq. Estas doentes poderão constituir um grupo considerado de maior risco a requerer uma vigilância mais apertada relativamente ao seu perfil metabólico, tendo em conta que a DM2, apesar de usualmente mais branda, pode ocorrer em idades mais precoces. É importante também ter conhecimento que estas doentes podem apresentar, muitas vezes, DM1 e outras condições autoimunes associadas. Portanto, é essencial reconhecer o risco aumentado de diabetes na ST, já que se sabe que a mortalidade cardiovascular nestas doentes se encontra aumentada ${ }^{19}$.

\section{Tireoidite de Hashimoto}

O diagnóstico de $\mathrm{TH}$ baseia-se na demonstração de anticorpos circulantes contra antígenos tireoidianos (principalmente tiroperoxidase e tireoglobulina) e ecogenicidade reduzida no ecograma tireoidiano em paciente com características clínicas adequadas ${ }^{20}$.

O tratamento permanece sintomático e com base na administração de hormônios tireoidianos sintéticos para corrigir o hipotireoidismo conforme necessário. A cirurgia é realizada quando o bócio é grande o suficiente para causar compressão significativa das estruturas cervicais circundantes, ou quando algumas áreas da glândula tireóide imitam as características de um nódulo cuja citologia não pode ser determinada como benigna ${ }^{20}$. Porém, segundo Heggie et Al. (2017), a tireoidectomia pode ser considerada em pacientes com sintomas de pressão significativos com diagnóstico de $\mathrm{TH}$, independentemente do tamanho aparente do bócio ${ }^{21}$.

Embora os relatos ou queixas de $\mathrm{TH}$ dolorosas estejam presentes na literatura, é importante ressaltar que somente 20 casos foram relatados do ano 2000 até agora. A condição, no entanto, é clinicamente relevante porque a dor no pescoço foi relatada como refratária tanto aos esteróides quanto a outros medicamentos analgésicos, sendo a tireoidectomia o único tratamento efetivo para o alívio da dor nesses pacientes ${ }^{22}$.

Em estudo realizado por Azizi et Al. (2014), houve associação de câncer de tireóide (CT) com a concentração de anticorpos antitireoglobulina sérica ( $\operatorname{TgAb}$ ) aumentada e idade <45 anos. Uma concentração elevada de TgAb no soro foi encontrada em $10,2 \%$ dos pacientes (182 de 1790) com nódulos benignos em comparação com $20,6 \%$ dos pacientes (48 de 233) com nódulos malignos. TgAb e TSH $\geq 1 \mu \mathrm{UI} / \mathrm{mL}$

foram preditores significativos de CT na análise multivariada controlando a idade e o sexo. CT não foi associado com concentrações séricas de anticorpo da peroxidase da tireóide (TPOAb). Em pacientes com nódulos tireoidianos, a concentração sérica elevada de TgAb e TSH $\geq 1 \mu \mathrm{UI} / \mathrm{mL}$ são preditores independentes para CT. A associação entre TH e CT é específica do anticorpo ${ }^{23}$. Portanto, a TH continua a ser uma doença complexa e em constante expansão, de patogênese desconhecida, e que aguarda prevenção ou novas formas de tratamento ${ }^{20}$.

\section{Doença de Crohn}

A patogênese da DC, uma doença inflamatória do intestino, envolve interações entre o genoma do hospedeiro, microbiota gastrointestinal e sistema imunológico da mucosa. Os estímulos iniciais e perpetuantes para a desregulação imune na DII não são explicados completamente por predisposição genética; outros fatores modificadores devem estar presentes ${ }^{24}$.

A comparação de microbiomas entre pacientes com DC com e sem exposição antibiótica indica que o uso de antibióticos amplifica a disbiose microbiana associada à DC. A comparação dos sinais microbianos entre o íleo, o reto e as amostras fecais indica que, neste estágio inicial da doença, avaliar o microbioma associado à mucosa retal oferece potencial exclusivo para o diagnóstico conveniente e precoce de $\mathrm{DC}^{25}$

Segundo Wright et Al. (2015), Alterações na composição e função microbiana foram descritas, embora ainda não seja estabelecido um papel causador. Estudos longitudinais maiores, prospectivos e longitudinais são necessários com interrogação profunda do microbioma se for determinada a causalidade, e a manipulação microbiana refinada deve emergir como uma terapia focada ${ }^{26}$.

Atualmente, tem-se apenas uma compreensão primitiva de como o microbioma intestinal influencia o sistema imunológico do hospedeiro. Com uma doença complexa, como a DII, ainda mais importante do que a doença de condução patogênica são os micróbios protetores que são 
importantes para o estabelecimento da homeostase entre o intestino e o microbioma. Em última análise, precisa-se obter uma melhor compreensão do papel das vias hospedeiromicróbio na manutenção da homeostase, a fim de proporcionar o maior valor para pacientes terapeuticamente ${ }^{27}$.

Estudos recentes mostram que o tratamento de acordo com o risco clínico de recorrência, com colonoscopia precoce e aumento do tratamento para a recorrência, é melhor do que a terapia convencional de drogas única para a prevenção da recorrência da DC pós-operatória. A supressão imune seletiva, ajustada para a recorrência precoce, em vez de uso rotineiro, leva ao controle da doença na maioria dos pacientes. Os fatores de risco clínicos prevêem recorrência, mas os pacientes com baixo risco também precisam de monitoramento. A remissão antecipada não exclui a necessidade de monitoramento contínuo ${ }^{28}$.

Como observado neste relato, duas filhas de um casal hígido manifestaram doenças autoimunes, as quais têm incidência aumentada na ST. Doenças endócrinas autoimunes possuem mecanismos complexos com a participação de vários fatores, como a susceptibilidade genética, eventos ambientais e resposta autoimune, porém, neste caso, não se encontrou história familiar positiva além da geração estudada.

\section{COMENTÁRIOS FINAIS}

Outros estudos familiares, como este que se propõe, com múltiplos membros acometidos, poderão identificar associações cada vez mais consistentes entre essas doenças, além daquelas ainda não suspeitadas.

\section{REFERÊNCIAS BIBLIOGRÁFICAS}

1. Larsen $T$, Gravholt $\mathrm{CH}$, Tillebeck $A$, Larsen $\mathrm{H}$, Jensen $\mathrm{MB}$, Nielsen $\mathrm{J}$, et al. Parental origin of the $\mathrm{X}$ chromosome, $\mathrm{X}$ chromosome mosaicism and screening for "hidden" $Y$ chromosome in $45, X$ Turner syndrome ascertained cytogenetically. Clin Genet. 1995; 48:6-11.

2. Nielsen J, Wohlert M. Chromosome abnormalities found among 34,910 newborn children: results from a 13-year incidence study in Arhus, Denmark. Hum Genet. 1991; 87:81-3.

3. Medeiros, Carla C.M. et al. Evidências de doença tireóidea auto-imune crônica subclínica em portadoras da Síndrome de Turner. Arq Bras Endocrinol Metab, São Paulo. 2007;51(3): $401-$ 409.

4. Mortensen KH, Cleeman L, Hjerrild BE, Nexo E, Loc H, Jeppesen $E M$, et al. Increased prevalence of autoimmunity in Turner syndromeinfluence of age. Clin Exp Immunol. 2009; 156:205 10.

5. Gravholt $\mathrm{CH}$. Clinical practice in Turner syndrome. Nat Clin Pract Endocrinol Metab. 2005; 1:41-52.

6. Sutton EJ, Mcinerney-Leo A, Bondy CA, Gollust SE, King D, BIESECKER B. Turner syndrome: four challenges across the lifespan. Am J Med Genet A. 2005;139A (2):57-66.

7. Fernandes, Ana Paula M. et al. Como entender a associação entre o sistema HLA e as doenças autoimunes endócrinas. Arq Bras Endocrinol Metab, São Paulo. 2003; 47(5): 601-611.

8. Medeiros CCM, Marini SHVL, Baptista MTM, Guerra JR G, Maciel-Guerra AT. Turner's syndrome and thyroid disease: a transverse study of pediatric patients in Brazil. J Pediatr Endocrinol Metabol. 2000; 13:357-62.
9. Gassull MA, Fernandez-Banares F, Cabré E, Esteves-ComAS M. Enteral nutrition in inflammatory bowel disease. In: Rombeau $\mathrm{JL}$, Rolandelli RH, editors. Clinical nutrition: enteral and tube feeding. Philadelphia: W.B. Saunders. 1997; 403-16.

10. Magro, F. Reflexoes sobre a etiopatogenia. Cadernos De Formação Em Gastrenterologia. Serviço de Gastrenterologia, Hospital de São João, Porto, Portugal. 2007; 14: 78-80.

11. Lennard-Jones JE, Longmore AJ, Newell AC, Wilson CW, Jones FA. An assessment of prednisone, salazopyrin, and topical hydrocortisone hemisuccinate used as out-patient treatment for ulcerative colitis. Gut. 1960; 1: 217-22.

12. Hanauer SB, Feagan BG, Lichtenstein GR, Mayer LF, Schreiber S, Colombel JF, Rachmilewitz D, Wolf DC, Olson A, Bao W, Rutgeerts. Maintenance infliximab for Crohn's disease: the ACCENT I randomised trial. Lancet. 2002; 359: 1541-9.

13. Farrant M, Mason JC, Wong NA, Longman RJ. Takayasu's arteritis following Crohn's disease in a young woman: Any evidence for a common pathogenesis? World J Gastroenterol. 2008; 14: 4087-90.

14. Hook EB, Warburton D. Turner syndrome revisited: review of new data supports the hypothesis that all viable $45, X$ cases are cryptic mosaics with a rescue cell line, implying an origin by mitotic loss. Hum Genet 2014; 133: 417-24.

15. Jorgensen KT, Rostgaard K, Bache I, Biggar RJ, Nielsen NM, Tommerup $\mathrm{N}$, et al. Autoimmune diseases in women with Turner's syndrome. Arthritis Rheum. 2010; 62(3): 658-66.

16. Mortensen $\mathrm{KH}$, Cleemann L, Hjerrild BE, Nexo E, Locht $H$, Jeppesen EM, et al. Increased prevalence of autoimmunity in Turner syndrome-influence of age. Clin Exp Immunol. 2009; 156(2): 205-10.

17. Pessach IM, Notarangelo LD: X-linked primary immunodeficiencies as a bridge to better understanding $X$ chromosome related autoimmunity. J Autoimmun 2009; 33:1724.

18. Stochholm K, Juul S, Juel K, Naeraa RW, Gravholt $\mathrm{CH}$. Prevalence, incidence, diagnostic delay, and mortality in Turner syndrome. J Clin Endocrinol Metab. 2006; 91(10): 3897-902.

19. Pereira JS, Bastos M, Saraiva J, Carolina M, Dantas R, Coelho D, et Al. Síndrome de Turner e diabetes mellitus - revisão da literatura a propósito de 4 casos. Rev Port Endocrinol Diabetes Metab. 2016; 11(2): 240-245.

20. Caturegli $P$, Remigis $A$, Rose NR. Hashimoto thyroiditis: Clinical and diagnostic criteria. Autoimmunity Reviews 13 (2014) 391397.

21. Heggie K, Yeung M, Grodski S, Lee J, Serpell J. Total thyroidectomy for pressure symptoms in patients with Hashimoto's thyroiditis. ANZ J Surg. 2017: 1-4.

22. Rotondi M, Capelli V, Locantore P, Pontecorvi A, Chiovato L. Painful Hashimoto's thyroiditis: myth or reality? J Endocrinol Invest. 2017; 40(8): 815-818.

23. Azizi G, Keller JM, Lewis M, Piper K, Puett D2, Rivenbark KM, Malchoff CD. Association of Hashimoto's thyroiditis with thyroid cancer. Endocr Relat Cancer. 2014; 21(6): 845-52.

24. Barclay AR, Morrison DJ, Weaver LT. What is the role of the metabolic activity of the gut microbiota in inflammatory bowel disease? Probing for answers with stable isotopes. J Pediatr Gastroenterol Nutr. 2008; 46: 486-495.

25. Gevers D, Kugathasan S, Denson LA, Vázquez-Baeza $Y$, Van Treuren $\mathrm{W}$, Ren $\mathrm{B}$ et Al. The treatment-naive microbiome in new-onset Crohn's disease. Cell Host Microbe. 2014; 15(3): 382-392.

26. Wright EK, Kamm MA, Teo SM, Inouye M, Wagner J, Kirkwood CD. Recent advances in characterizing the gastrointestinal microbiome in Crohn's disease: a systematic review. Inflamm Bowel Dis. 2015;21(6): 1219-28. 
27. Gevers D, Kugathasan S, Knights D, Kostic AD, Knight R, Xavier RJ. A Microbiome Foundation for the Study of Crohn's Disease. Cell Host Microbe. 2017; 21(3): 301-304.

28. De Cruz P, Kamm MA, Hamilton AL, Ritchie KJ, Krejany EO, Gorelik A et Al. Crohn's disease management after intestinal resection: a randomised trial. Lancet. 2015; 385(9976): 140617. 